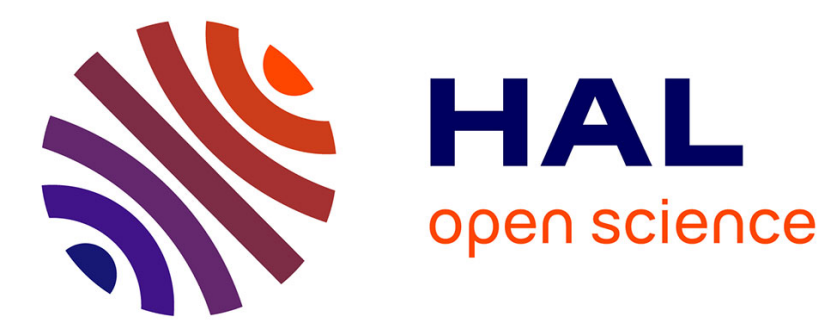

\title{
Report of false positives when using zymography to assess peptidoglycan hydrolytic activity of an endopeptidase with multiple LysM domains
}

\author{
Jaslyn E.M.M. Wong, Mickael Blaise
}

\section{- To cite this version:}

Jaslyn E.M.M. Wong, Mickael Blaise. Report of false positives when using zymography to assess peptidoglycan hydrolytic activity of an endopeptidase with multiple LysM domains. Biochimie, 2020, 177, pp.25-29. 10.1016/j.biochi.2020.07.014 . hal-02988315

\section{HAL Id: hal-02988315 \\ https://hal.science/hal-02988315}

Submitted on 5 Nov 2020

HAL is a multi-disciplinary open access archive for the deposit and dissemination of scientific research documents, whether they are published or not. The documents may come from teaching and research institutions in France or abroad, or from public or private research centers.
L'archive ouverte pluridisciplinaire HAL, est destinée au dépôt et à la diffusion de documents scientifiques de niveau recherche, publiés ou non, émanant des établissements d'enseignement et de recherche français ou étrangers, des laboratoires publics ou privés. 


\section{Biochimie}

\section{Report of false positives when using zymography to assess peptidoglycan hydrolytic activity of an endopeptidase with multiple LysM domains. \\ --Manuscript Draft--}

\begin{tabular}{|c|c|}
\hline Manuscript Number: & BIOCHI-D-20-00474R1 \\
\hline Article Type: & Short Communication \\
\hline Section/Category: & Regular issue \\
\hline Keywords: & Bacillus subtilis; LysM; zymography; peptidoglycan; NIpC/P60 endopeptidase \\
\hline Corresponding Author: & $\begin{array}{l}\text { Mickael Blaise, PhD } \\
\text { CNRS Délégation Languedoc-Roussillon } \\
\text { Montpellier, Languedoc-Roussillon FRANCE }\end{array}$ \\
\hline First Author: & Jaslyn Mei Mei Wong, PhD \\
\hline \multirow[t]{2}{*}{ Order of Authors: } & Jaslyn Mei Mei Wong, PhD \\
\hline & Mickael Blaise, PhD \\
\hline Manuscript Region of Origin: & FRANCE \\
\hline Abstract: & $\begin{array}{l}\text { Zymography is a widely used technique enabling visualization of in-gel } \\
\text { peptidase/protease hydrolytic activities. This technique is used to study the activity of } \\
\text { bacterial peptidoglycan (PG) hydrolytic enzymes named autolysins. Zymography is } \\
\text { particularly suited for PG autolysin characterization as bulk PG is notorious to work } \\
\text { with due to its highly insoluble nature. This recalcitrant property of PG therefore makes } \\
\text { the set-up of PG hydrolytic activity assay very challenging. In the course of studying } \\
\text { the catalytic activity of the CwIS protein, a D, L NlpC/P60 endopeptidase possessing } \\
\text { multiple LysM carbohydrate binding-domains from Bacillus subtilis, we observed a } \\
\text { potential artifact of the zymography technique. The generation of CwIS truncated } \\
\text { mutants impaired in their PG binding capacity presented lower apparent hydrolytic } \\
\text { activities on zymograms. Furthermore, a catalytically dead version of CwIS, or a CwIS } \\
\text { mutant that possesses only its LysM domains and no catalytic domain, maintained } \\
\text { similar apparent PG hydrolytic properties as wild-type CwIS on zymograms. } \\
\text { Additionally, a mutant harboring twelve mutations in the four LysM domains, previously } \\
\text { demonstrated to be unable to bind PG but has a similar net positive charge as the wild- } \\
\text { type protein presents also apparent activity on zymogram. We demonstrate in this } \\
\text { study that zymography results, which are meant to be interpreted in favor of apparent } \\
\text { PG hydrolytic activities, are instead reflecting impairment of gel staining probably due } \\
\text { to the very high net positive charge of the protein. }\end{array}$ \\
\hline Opposed Reviewers: & \\
\hline
\end{tabular}


Report of false positives when using zymography to assess peptidoglycan hydrolytic activity of an endopeptidase with multiple LysM domains.

\author{
Jaslyn E.M.M Wong ${ }^{1,+}$ and Mickaël Blaise ${ }^{1,2}$ \\ 1-Department of Molecular Biology and Genetics, Aarhus University, Gustav Wieds Vej 10, 8000, \\ Aarhus, Denmark \\ 2-Present address: Institut de Recherche en Infectiologie de Montpellier (IRIM), Université de \\ Montpellier, CNRS UMR 9004, 1919 route de Mende, 34293 Montpellier, France. \\ + Present address: MRC Laboratory of Molecular Biology, Cambridge CB2 0QH, United Kingdom
}

\begin{abstract}
Zymography is a widely used technique enabling visualization of in-gel peptidase/protease hydrolytic activities. This technique is used to study the activity of bacterial peptidoglycan (PG) hydrolytic enzymes named autolysins. Zymography is particularly suited for PG autolysin characterization as bulk PG is notorious to work with due to its highly insoluble nature. This recalcitrant property of PG therefore makes the set-up of PG hydrolytic activity assay very challenging. In the course of studying the catalytic activity of the CwlS protein, a D,L NlpC/P60 endopeptidase possessing multiple LysM carbohydrate binding-domains from Bacillus subtilis, we observed a potential artifact of the zymography technique. The generation of CwlS truncated mutants impaired in their PG binding capacity presented lower apparent hydrolytic activities on zymograms. Furthermore, a catalytically dead version of CwlS, or a CwlS mutant that possesses only its LysM domains and no catalytic domain, maintained similar apparent PG hydrolytic properties as wild-type CwlS on zymograms. Additionally, a mutant harboring twelve mutations in the four LysM domains, previously demonstrated to be unable to bind PG but has a similar net positive charge as the wild-type protein presents also apparent activity on zymogram. We demonstrate in this study that zymography results, which are meant to be interpreted in favor of apparent PG hydrolytic activities, are instead reflecting impairment of gel staining probably due to the very high net positive charge of the protein.
\end{abstract}




\section{Highlights}

-False positives peptidoglycan hydrolytic activities were revealed by zymography

-False positives zymography results do not reflect loss of peptidoglycan binding strength

-Zymography artifact is linked to the net protein charge 


\title{
Report of false positives when using zymography to assess peptidoglycan hydrolytic activity of an endopeptidase with multiple LysM domains.
}

\author{
Jaslyn E.M.M Wong ${ }^{1,+}$ and Mickaël Blaise ${ }^{1,2}$ \\ 1-Department of Molecular Biology and Genetics, Aarhus University, Gustav Wieds Vej 10, 8000, \\ Aarhus, Denmark \\ 2-Present address: Institut de Recherche en Infectiologie de Montpellier (IRIM), Université de \\ Montpellier, CNRS UMR 9004, 1919 route de Mende, 34293 Montpellier, France. \\ + Present address: MRC Laboratory of Molecular Biology, Cambridge CB2 0QH, United Kingdom
}

To whom correspondence should be addressed:

Mickaël Blaise: mickael.blaise@ irim.cnrs.fr; phone: +33 (0)434359447

Jaslyn E.M.M. Wong: jwong@mrc-lmb.cam.ac.uk; phone: +44 (0)1223 267625

\section{Highlights}

-False positives peptidoglycan hydrolytic activities were revealed by zymography -False positives zymography results do not reflect loss of peptidoglycan binding strength -Zymography artifact is linked to the net protein charge

\begin{abstract}
Zymography is a widely used technique enabling visualization of in-gel peptidase/protease hydrolytic activities. This technique is used to study the activity of bacterial peptidoglycan (PG) hydrolytic enzymes named autolysins. Zymography is particularly suited for PG autolysin characterization as bulk PG is notorious to work with due to its highly insoluble nature. This recalcitrant property of PG therefore makes the set-up of PG hydrolytic activity assay very challenging. In the course of studying the catalytic activity of the CwlS protein, a D,L NlpC/P60 endopeptidase possessing multiple LysM carbohydrate binding-domains from Bacillus subtilis, we observed a potential artifact of the zymography technique. The generation of CwlS truncated mutants impaired in their PG binding capacity presented lower apparent hydrolytic activities on zymograms. Furthermore, a catalytically dead version of CwlS, or a CwlS mutant that possesses only its LysM domains and no catalytic domain, maintained similar apparent PG hydrolytic properties as wild-type CwlS on zymograms. Additionally, a mutant harboring twelve mutations in the four LysM domains, previously demonstrated to be unable to bind PG but has a similar net positive charge as the wild-type protein presents
\end{abstract}


also apparent activity on zymogram. We demonstrate in this study that zymography results, which are meant to be interpreted in favor of apparent PG hydrolytic activities, are instead reflecting impairment of gel staining probably due to the very high net positive charge of the protein.

Keywords: Bacillus subtilis; LysM; zymography; peptidoglycan; NlpC/P60 endopeptidase.

\section{Introduction}

Bacteria are shielded from environmental stresses by a cell wall, which contains a peptidoglycan $(\mathrm{PG})$ layer. $\mathrm{PG}$ is a three-dimensional mesh-like structure composed of repeating units of MurNAc and GlcNAc. Long polymers containing MurNAc-GlcNAc repeats are linked to each other by peptide stems, enabling the formation of a complex 3D network [1]. Although the structure of PG is very rigid, it has also to be highly dynamic during bacterial cell division processes, notably, to facilitate the separation of the two daughter cells and neosynthesis of PG [2-4]. During these phases, PG remodeling is mediated by autolysins that are a group of PG-cleaving enzymes with different substrate specificities. These enzymes either cleave the carbohydrate backbone, the peptide stem, or the bond between MurNAc and the peptide stem or the peptide stem at specific positions. The complex and insoluble nature of PG renders the development of activity assays to characterize autolysins very challenging [5]. Zymography, a widely used technique allowing in-gel visualization of substrate hydrolysis, helps to overcome this challenge [6]. The principle of zymography is based on the migration of a protein of interest into a native or non-denaturing electrophoresis gel in which the substrate is incorporated. The substrate hydrolysis can be then detected where the protein endmigrates [6]. It has been extensively used to study matrix metalloproteases in eukaryotes [7] but also to study bacterial PG hydrolytic enzymes.

To study autolysin activities by zymography, bacterial cell wall or bulk PG can be incorporated in the polyacrylamide gels. After gel electrophoresis, in native or denaturing conditions of the purified protein of interest or even crude protein extracts, the PG is stained and any presence of a white zone of clearing corresponding to the end migration of the protein would indicate hydrolysis of PG. When separation is performed under denaturing conditions, the gel is extensively washed to remove sodium dodecyl 
sulfate (SDS) and the protein is refolded slowly by placing the gel into a renaturation buffer prior to staining.

We studied the PG binding properties of the CwlS protein from Bacillus subtilis [8]. This enzyme is a NlpC/P60 D,L-endopeptidase capable of cleaving the PG peptide stem between the $2^{\text {nd }}$ and $3^{\text {rd }}$ amino acids. CwlS possesses a repeat of four LysM domains that are known binding modules of chitin and MurNAc-GlcNAc polymers [9]. The CwlS encoding gene is essential for daughter cell separation in B. subtilis [10]. In the course of characterizing CwlS and CwlS mutants by biochemical approaches, we noticed that the apparent hydrolytic activity was not correlated to the catalytic properties observed on the zymograms. In this study, we, therefore, report these observations with the aim of drawing attention to the potential misinterpretation of results of this widely used technique.

\section{Materials and methods}

\subsection{Cloning, Protein expression and Purification}

CwlS wild-type (CwlS_FL), 3LysM-Cata, 2LysM-Cata, 1LysM-Cata, Cata, FL_Mut and 4LysM constructs were essentially cloned and purified as previously described [8]. The CwlS-C326A mutant was generated with the CCTGCCGGTTTTGATGCCAGCGGCTTCATT forward primer and AATGAAGCCGCTGGCATCAAAACCGGCAGG reverse primer using the QuikChange Lightning Site-Directed Mutagenesis Kits (Agilent Technologies) according to the manufacturer's instructions and using the CwlS-FL gene as a template. This mutant was expressed and purified as similar as wild-type CwlS.

\subsection{Zymography}

To purify B. subtilis cell wall, B. subtilis cells (ATCC 6056) were grown in $100 \mathrm{ml} \mathrm{LB}$ media overnight at $30^{\circ} \mathrm{C}$. The cells were centrifuged at $10,500 \mathrm{~g}$ for $15 \mathrm{~min}$, and washed twice with $50 \mathrm{mM}$ Phosphate $\mathrm{pH}$ 7.5. The cell lysate was then centrifuged at 30,000 $\mathrm{g}$ for $20 \mathrm{~min}$, resuspended in $0.5 \% \mathrm{SDS}$, and incubated at $60{ }^{\circ} \mathrm{C}$ for $30 \mathrm{~min}$. The cells were centrifuged at 30,000 $\mathrm{g}$ for $20 \mathrm{~min}$ and washed with water. This step was repeated five times. Extracted B. subtilis cell wall was digested with $100 \mu \mathrm{g}$ ml-1 trypsin in $1 \mathrm{M}$ Tris$\mathrm{HCl} \mathrm{pH} 7$ overnight at $37^{\circ} \mathrm{C}$. The cell wall was then centrifuged at 30,000 g for $20 \mathrm{~min}$ and washed with $1 \mathrm{M}$ Tris- $\mathrm{HCl} \mathrm{pH}$ 7. This step was repeated once with $1 \mathrm{M}$ Tris- $\mathrm{HCl}$ 
$\mathrm{pH} 7$ and $1 \mathrm{M} \mathrm{NaCl}$, once with $1 \mathrm{M}$ Tris- $\mathrm{HCl} \mathrm{pH} 7$, and six times with water. Zymography gels were prepared by adding $0.4 \%$ (w/w) cell wall extract to $15 \%$ SDSPAGE. $20 \mu \mathrm{g}$ of protein in loading buffer were loaded in each lane. $10 \mu \mathrm{g}$ BSA and 0.5 $\mu \mathrm{g}$ lysozyme were added as negative and positive control respectively. After SDSPAGE, the gels were incubated in $50 \mathrm{mM}$ Tris-HCl pH 8 buffer for 48 hours, then stained with $0.1 \%(\mathrm{w} / \mathrm{v})$ methylene blue diluted in $0.01 \% \mathrm{KOH}$.

\subsection{Assessment of the endopeptidase activity using turbidity activity assay}

The endopeptidase activity assay was performed as previously described [8].

\subsection{Overall protein net charge determination}

The overall net charges of the protein variants at $\mathrm{pH} 7$ were determined using this server: https://pepcalc.com/protein-calculator.php. The protein charges were calculated according to the following formula: $Z=\sum_{i} N_{i} \frac{10^{p K a_{i}}}{10^{p H}+10^{p K a_{i}}}-\sum_{j} N_{j} \frac{10^{p H}}{10^{p H}+10^{p K a_{j}}}$ where $N_{i}$ indicates the number, and $\mathrm{pKa}_{i}$ the $\mathrm{pKa}$ values, of the $\mathrm{N}$-terminus and the side chains of positively charged amino acids: Arginine, Lysine, and Histidine. The $j$-index relates to the C-terminus and the negatively charged residues: Aspartic Acid, Glutamic Acid as well as Cysteine and, Tyrosine amino acids.

\section{Results and discussion}

The CwlS study was undertaken to reveal the role of the $\mathrm{N}$-terminal region of the protein containing multiple LysM domains (Figure 1A). The LysM domain is a known PG binding domain [9], so we initially performed a study to assess if the number of LysM repeats was conferring substrate-binding strength. We showed that the CwlS affinity towards PG and chitin polymers is indeed influenced by the numbers of LysM repeats which cooperated to bind these long polymers [8]. The dissociation constants $\left(K_{\mathrm{d}}\right)$ determined for chitin and MurNAc-GlcNAc polymers using the Microscale Thermophoresis method demonstrated that the $K_{\mathrm{d}}$ increased alongside LysM deletion (Figure 1A) [8]. A positive correlation between carbohydrate affinity and the number of LysM domains was also confirmed in studies using NlpC/P60 from Thermus thermophilus [11] and the AtlA autolysin from Enterococcus faecalis [12]. 
In this study, we assessed if the deletion of LysM modules could influence the denaturing gels containing bulk PG from B. subtilis. Full-length wild-type CwlS protein (CwlS-FL) seemed capable of cleaving PG as a white zone of clearing corresponding to the end-migration position of the protein was observed after methylene blue staining of zymography gels (Figure 1B). We then assessed the PG-cleaving abilities of single to four LysM deletion in CwlS mutants and noticed that the more impaired the CwlS mutants were in their PG binding capacities the lower their abilities were to form white zones of clearing on zymogram (Figure 1A and 1B). It appeared that CwlS-FL and 3LysM-Cata proteins that have similar dissociation constants for PG, cleaved PG the most efficiently as indicated by most distinct zones of clearing in zymograms compare with 2LysM-Cata, 1LysM-Cata and Cata mutants that were strongly impaired in PG binding. These mutants presented less pronounced zones of clearing. The Cata mutant that did not possess any PG binding module produced barely detectable zone of clearing. This observation could have been considered in perfect agreement with our previously published results showing that these mutants were impaired in their capacity to hydrolyze Bacillus subtilis culture which could be explained by their loss of ability to anchor to the cell wall [8].

Counter-intuitively, a protein mutant $(4 \mathrm{LysM})$ that did not contain any catalytic domain but only the four LysM domains also generated zones of clearing on zymograms as pronounced as the wild-type protein, CwlS-FL (Figure 2A). Puzzled by the apparent PG hydrolytic activity of a CwlS variant that does not actually contain a catalytic domain, we further generated a catalytically dead mutant (CwlS-C326A) by site-directed mutagenesis of a catalytic cysteine that is present in the active site of the protein; this cysteine residue is strictly conserved in NlpC/P60 endopeptidases and is essential for PG hydrolysis [13-15]. Surprisingly, the CwlS-C326A mutant also generated a white zone of clearing in zymograms with equal intensity as the wild-type protein (Figure 2A).

To rule out that neither the 4LysM nor the CwlS-C326A mutant possessed PG hydrolytic properties, we assessed their activities on exponentially growing planktonic B. subtilis cultures. We added the purified proteins to B. subtilis cultures and monitored the cell density over $140 \mathrm{~min}$. If the proteins are active, they should lyse the cells, and this is reflected by a drop in turbidity readings. CwlS-FL and the positive control, lysozyme, hydrolyzed B. subtilis as indicated by a drop in optical density of $\sim 0.3$ units (Figure 2B) in about 2 hours. In contrast, an increase in optical cell density was 
observed for cells treated with the 4LysM and CwlS-C326A mutants, similar to the negative controls, BSA or protein buffer. The inability of the 4LysM and CwlS-C326A mutants to hydrolyze B. subtilis cultures (Figure 2B) strongly indicated that their corresponding zones of clearing on the zymograms do not reflect the enzymatic activity. One could consider in such a case, that zymography was in fact reflecting PG binding event and not PG hydrolysis.

Interestingly, the ChiZ protein from Mycobacterium tuberculosis that is part of the divisome and possesses a single LysM domain in its C-terminus also gave positive results on a zymogram albeit not being confirmed by other methods to be a PG hydrolase [16]. The authors elegantly proposed that the high net positive charge of ChiZ is in fact impairing toluidine blue coloration. Toluidine blue is indeed a positively charged compound that stains negatively charged PG, thus positively charged protein would repulse toluidine blue. The authors further demonstrated that the highly positively charged $\mathrm{CrgA}$ protein with no PG-related function also displayed apparent PG hydrolytic activity on zymogram [16].

We therefore calculated the net charge of all our CwlS variants (Figure 1A). With the exception of the Cata mutant with a net charge of 5 , the other proteins have all very high net positive charges, from 12 to 33 as compared with ChiZ that has a net charge of 10. The intensity of the zones of clearing of the different $\mathrm{CwlS}$ version is indeed correlated with net positive charges. The Cata mutant with a net charge of +5 generates indeed a rather weak zone of clearing compare with the CwlS-FL that has a net charge of +33 . To completely rule out the possibility that the positive zymogram result observed is not due to the loss of peptidoglycan binding strength but is rather related to the net positive charge of the protein, we included an additional mutant (FLMut) in our zymography assays. The FL-Mut (Figure 1A) presents twelve mutations, with three residues in each of the four LysM domains mutated into Ala. The mutagenesis was driven by docking studies which revealed amino acids with side chains that could potentially interact with carbohydrates as described in [8]. This mutant is highly impaired in its capacity to bind PG but very importantly has a similar net positive charge as the wild-type protein (Figure 1A). As assessed on zymogram (Figure 2C), this FL-Mut also resulted in a zone of clearance that was as pronounced as the CwlS-FL protein and the CwlS-C326A catalytic mutant that both possess similar net positive charges. Furthermore, the FL_Mut apparent activity is more pronounced than the CwlS variant that possessed only a catalytic domain and has a much lower net positive charge 
(Figure 2C). Altogether our data provides compelling evidence that the apparent activity of all the CwlS variants presented in this study, as assessed by zymography, is not correlated to PG binding strength but more probably to the net positive charge of the protein.

Kohler et al,, reported similar zymogram false positive when studying the autolysin AtlA from Neisseria gonorrhoeae [17]. The authors noticed that a catalytic dead mutant had apparent PG hydrolysis activity on zymogram although this mutant was, in fact, inactive in PG degradation assay or cell lysis assays [17]. The authors proposed that the protein was able to bind PG, therefore avoiding the PG coloration by toluidine blue. We calculated the net charge of AtlA, and noticed that it also has a high positive value of 10. Altogether, the studies of ChiZ, AtlA and CwlS, all possessing high net positive charges are therefore in favor of the hypothesis of impairment of coloration due to charges repulsion [16].

We hereby demonstrated that zymography could trigger false-positives when probing for PG hydrolysis activities of a multiple containing LysM protein. This communication is nonetheless not intended to discredit the use of zymography, which has been extensively and successfully used in autolysin hydrolysis assays for decades, but to create awareness that the data should be interpreted with caution. Zymography has in most cases been proven to be trustworthy as hydrolytic activities have successfully been confirmed by additional techniques. To avoid such artifact, Escobar et al., proposed to compare the results of gels revealed with and without a refolding step [16]. Alternatively or in complement, we would advise to determine the net charge of the protein of interest, and if possible, to assess the activities of a catalytic dead version alongside a wild-type version of the protein when performing zymography assessments, and most certainly to confirm the PG hydrolase activity by another method [5].

\section{Acknowledgements}

We would like to thank Pr Jens Stougaard for constant support.

\section{Funding}

Part of this work was funded by the Danish National Research Foundation grant No. DNRF79

\section{Declarations of interest}


All authors declare no conflict of interest.

\section{Authors contribution}

JW and MB designed research. JW performed experiments. JW and MB analyzed data.

MB supervised research. MB and JW wrote the manuscript.

\section{References}

[1] Meroueh SO, Bencze KZ, Hesek D, Lee M, Fisher JF, Stemmler TL, et al. Threedimensional structure of the bacterial cell wall peptidoglycan. Proc Natl Acad Sci U S A 2006;103:4404-9. https://doi.org/10.1073/pnas.0510182103.

[2] Typas A, Banzhaf M, Gross CA, Vollmer W. From the regulation of peptidoglycan synthesis to bacterial growth and morphology. Nat Rev Microbiol 2012;10:123-36. https://doi.org/10.1038/nrmicro2677.

[3] Vollmer W. Bacterial growth does require peptidoglycan hydrolases. Mol Microbiol 2012;86:1031-5. https://doi.org/10.1111/mmi.12059.

[4] Egan AJF, Errington J, Vollmer W. Regulation of peptidoglycan synthesis and remodelling. Nat Rev Microbiol 2020:1-15. https://doi.org/10.1038/s41579-0200366-3.

[5] Do T, Page JE, Walker S. Uncovering the activities, biological roles, and regulation of bacterial cell wall hydrolases and tailoring enzymes. J Biol Chem 2020;295:3347-61. https://doi.org/10.1074/jbc.REV119.010155.

[6] Vandooren J, Geurts N, Martens E, Van den Steen PE, Opdenakker G. Zymography methods for visualizing hydrolytic enzymes. Nat Methods 2013;10:211-20. https://doi.org/10.1038/nmeth.2371.

[7] GROSS J, LAPIERE CM. Collagenolytic activity in amphibian tissues: a tissue culture assay. Proc Natl Acad Sci U S A 1962;48:1014-22.

[8] Wong JEMM, Alsarraf HMAB, Kaspersen JD, Pedersen JS, Stougaard J, Thirup S, et al. Cooperative binding of LysM domains determines the carbohydrate affinity of a bacterial endopeptidase protein. FEBS J 2014;281:1196-208. https://doi.org/10.1111/febs.12698.

[9] Buist G, Steen A, Kok J, Kuipers OP. LysM, a widely distributed protein motif for binding to (peptido)glycans. Mol Microbiol 2008;68:838-47. https://doi.org/10.1111/j.1365-2958.2008.06211.X.

[10] Fukushima T, Afkham A, Kurosawa S-I, Tanabe T, Yamamoto H, Sekiguchi J. A new D,L-endopeptidase gene product, YojL (renamed CwlS), plays a role in cell separation with LytE and LytF in Bacillus subtilis. J Bacteriol 2006;188:5541-50. https://doi.org/10.1128/JB.00188-06.

[11] Wong JEMM, Midtgaard SR, Gysel K, Thygesen MB, Sørensen KK, Jensen KJ, et al. An intermolecular binding mechanism involving multiple LysM domains mediates carbohydrate recognition by an endopeptidase. Acta Crystallogr D Biol Crystallogr 2015;71:592-605. https://doi.org/10.1107/S139900471402793X.

[12] Mesnage S, Dellarole M, Baxter NJ, Rouget J-B, Dimitrov JD, Wang N, et al. Molecular basis for bacterial peptidoglycan recognition by LysM domains. Nat Commun 2014;5:4269. https://doi.org/10.1038/ncomms5269. 
[13] Xu Q, Sudek S, McMullan D, Miller MD, Geierstanger B, Jones DH, et al. Structural basis of murein peptide specificity of a gamma-D-glutamyl-1-diamino acid endopeptidase. Struct Lond Engl 1993 2009;17:303-13. https://doi.org/10.1016/j.str.2008.12.008.

[14] Xu Q, Mengin-Lecreulx D, Patin D, Grant JC, Chiu H-J, Jaroszewski L, et al. Structure-Guided Functional Characterization of DUF1460 Reveals a Highly Specific NlpC/P60 Amidase Family. Struct Lond Engl 1993 2014;22:1799-809. https://doi.org/10.1016/j.str.2014.09.018.

[15] Ruggiero A, Marasco D, Squeglia F, Soldini S, Pedone E, Pedone C, et al. Structure and functional regulation of RipA, a mycobacterial enzyme essential for daughter cell separation. Struct Lond Engl 1993 2010;18:1184-90. https://doi.org/10.1016/j.str.2010.06.007.

[16] Escobar CA, Cross TA. False positives in using the zymogram assay for identification of peptidoglycan hydrolases. Anal Biochem 2018;543:162-6. https://doi.org/10.1016/j.ab.2017.12.016.

[17] Kohler PL, Hamilton HL, Cloud-Hansen K, Dillard JP. AtlA functions as a peptidoglycan lytic transglycosylase in the Neisseria gonorrhoeae type IV secretion system. J Bacteriol 2007;189:5421-8. https://doi.org/10.1128/JB.00531-07.

\section{Figures legends}

\section{Figure 1: Determination of the peptidoglycan hydrolytic activity of CwlS mutant} by zymography

A-Schematic representation of the CwlS variants assessed in this study with the PGanchoring and catalytic domains indicated. The CwlS PG-anchoring domain corresponds to the LysM modules present in the protein. $\mathrm{N}-\mathrm{t}$ and $\mathrm{C}-\mathrm{t}$ stand for $\mathrm{N}$ terminus and $\mathrm{C}$-terminus respectively. *Indicates the dissociation constants $\left(K_{d}\right)$ previously determined by microscale thermophoresis using Staphylococcus aureus PG tetramers [8], \pm represents the standard deviation (with $95 \%$ confidence interval). N.d. indicates that binding was not detectable and n.t. indicates that binding was not tested. The isoelectric point (pI) and the overall net charges of the CwlS variants (without its peptide signal of secretion) at a $\mathrm{pH}$ of 7 are shown. The $\mathrm{pI}$ and the overall net charge at $\mathrm{pH} 7$ of the different protein variants were determined using this server: https://pepcalc.com/protein-calculator.php. B-Determination of the apparent PG hydrolytic activity of the different CwlS variants with one, two, three, or all LysM domains deleted. $\mathrm{M}$ indicates the protein molecular weight marker. Bovine serum albumin (BSA) and lysozyme were used as negative and positive controls, respectively. The dotted lines signify that the two gels are independent but were run simultaneously in the exact same conditions. Empty lanes in the gels were cropped. 
Figure 2: Assessment of the PG hydrolytic properties of CwlS mutants by zymography and upon incubation with planktonic $B$. subtilis culture

A-Zymography assessment of the apparent PG hydrolytic activity of wild-type CwlS and mutated versions devoid of a catalytic domain (4LysM) or containing a mutation in the catalytic domain (C326A) rendering it inactive. B-B. subtilis cell lysis capacities of the different CwlS variants. A drop of OD at $600 \mathrm{~nm}$ indicates cell lysis. The data is representative of three independent experiments. C- Comparison of the apparent PG hydrolytic activity of the FL_Mut variant versus CwlS_FL, CwlS_C326A and Cata mutants. 
Aigure1

PG anchoring domain

Catalytic domain

$K_{\mathrm{d}}(\mu \mathrm{M})$ for $\mathrm{PG}^{*}$

pI / Net charge

CwIS-FL

3LysM-Cata

2LysM-Cata

1LysM-Cata

Cata

4LysM

CwIS-C326A

FL-Mut

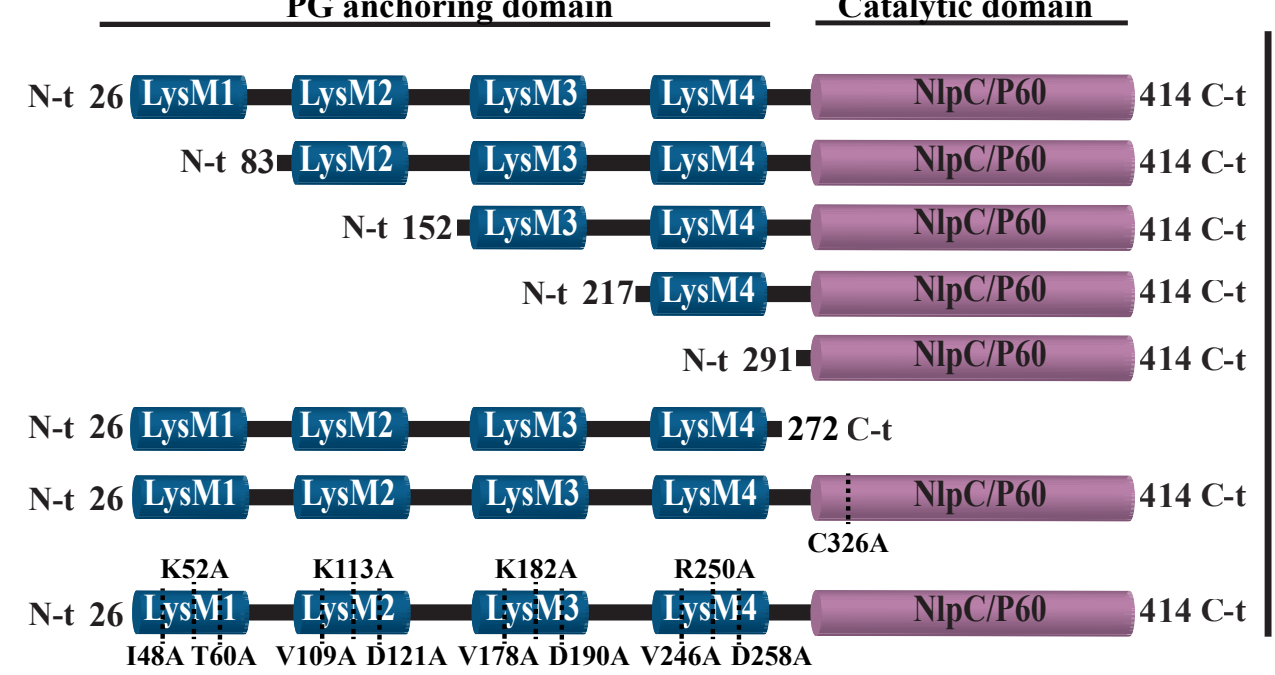

$10.1 \pm 2.1$

$9.1 \pm 1.7$

n.d.

n.d.

n.d.

$6.8 \pm 1.3$

n.t.

n.d.
10 / 33.2

$9.9 / 26.2$

9.9 / 19.1

$9.8 / 12.1$

9.4 / 5.1

$10.1 / 25.1$

$10 / 33.2$

$10 / 32.2$

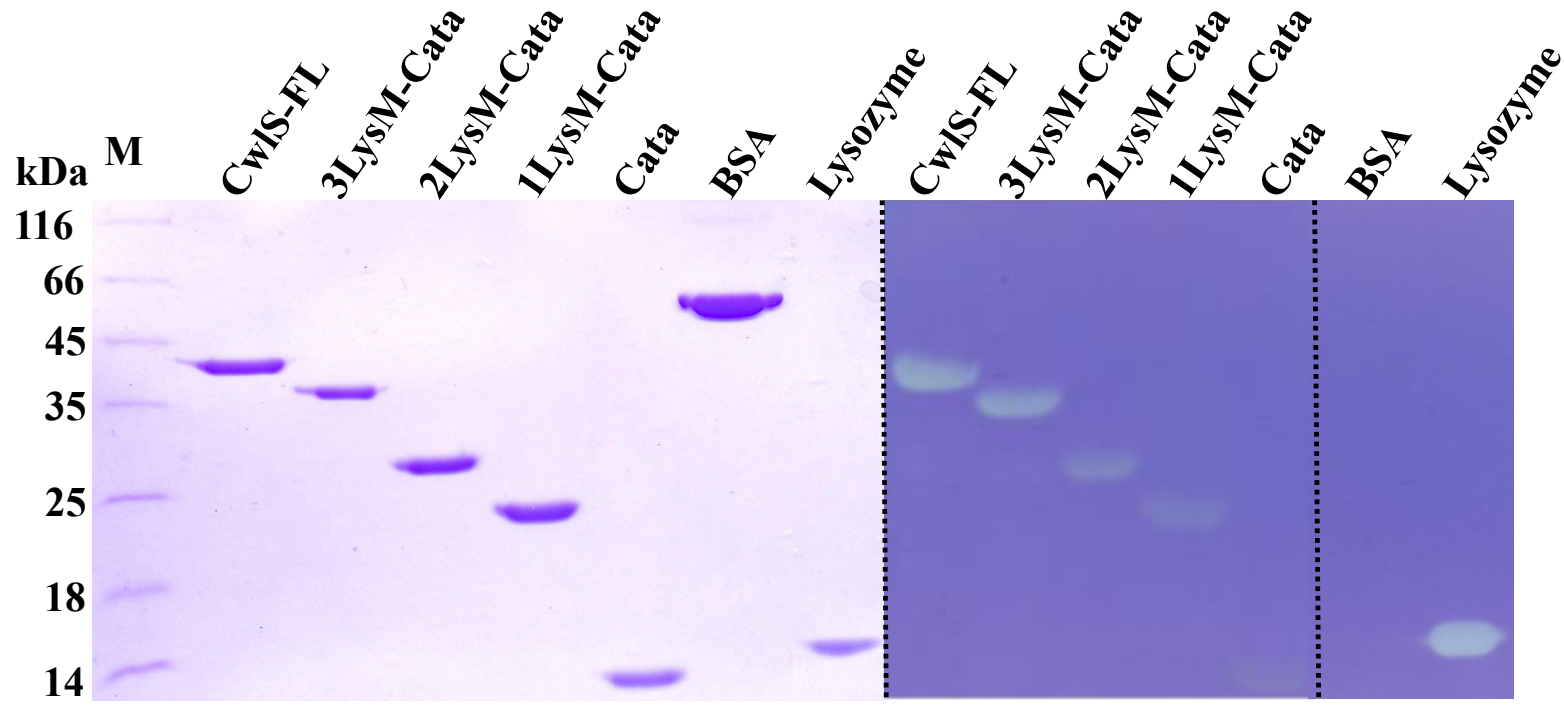

Coomassie stained 


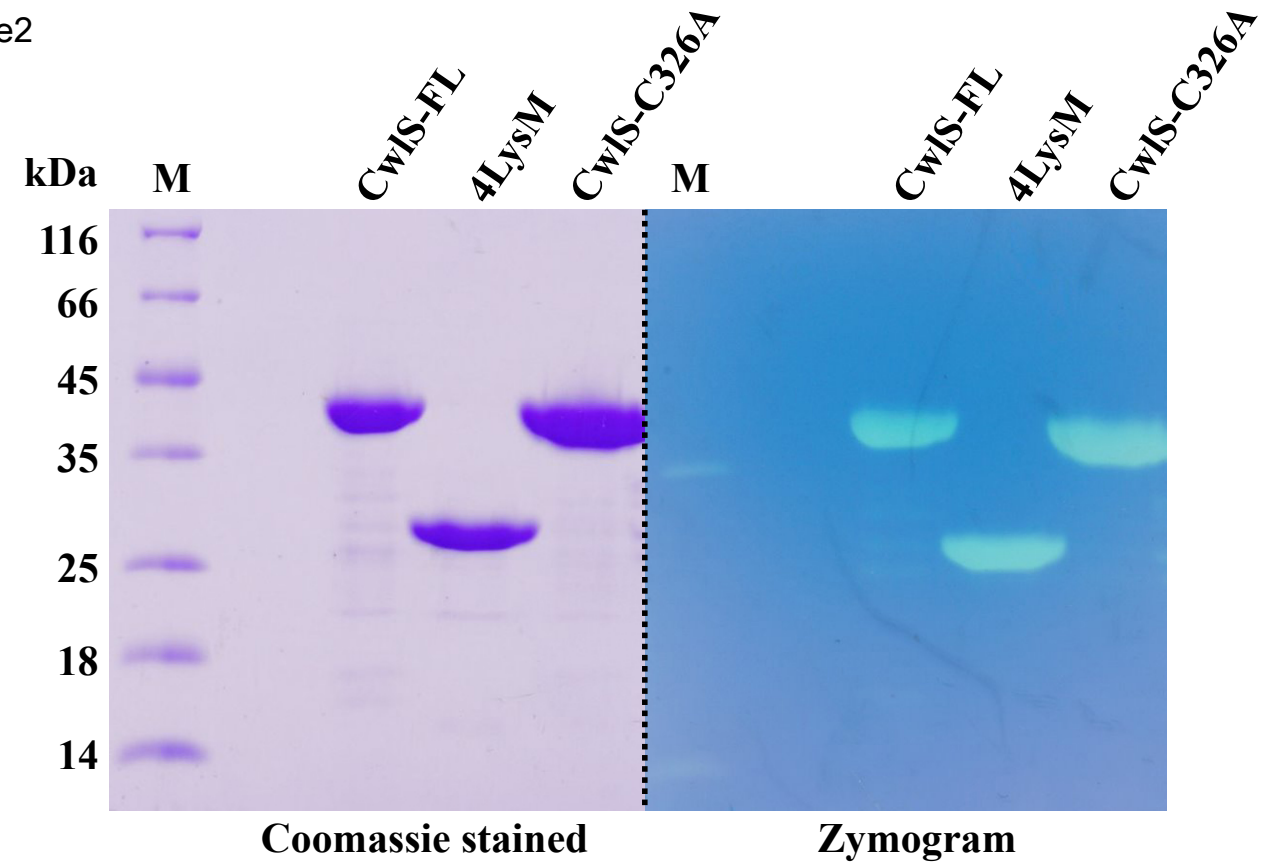

B

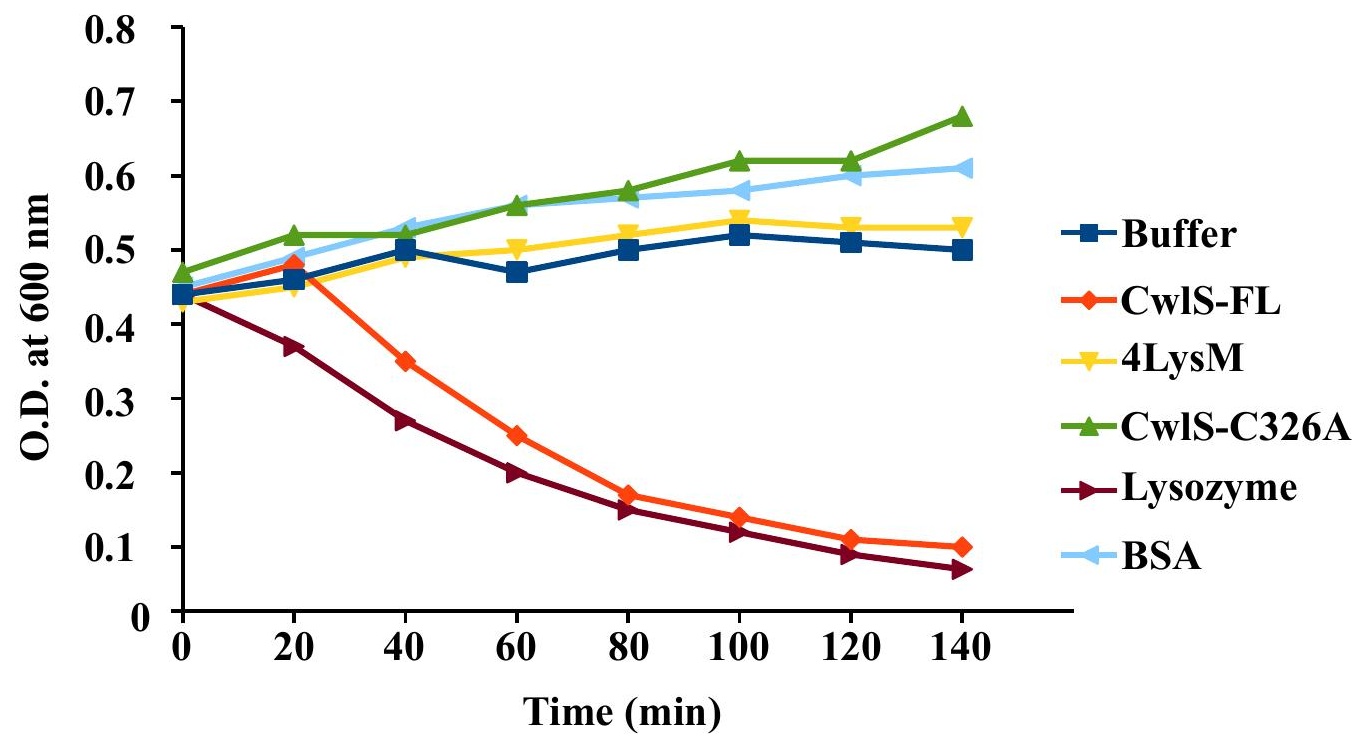

$\mathrm{C}$

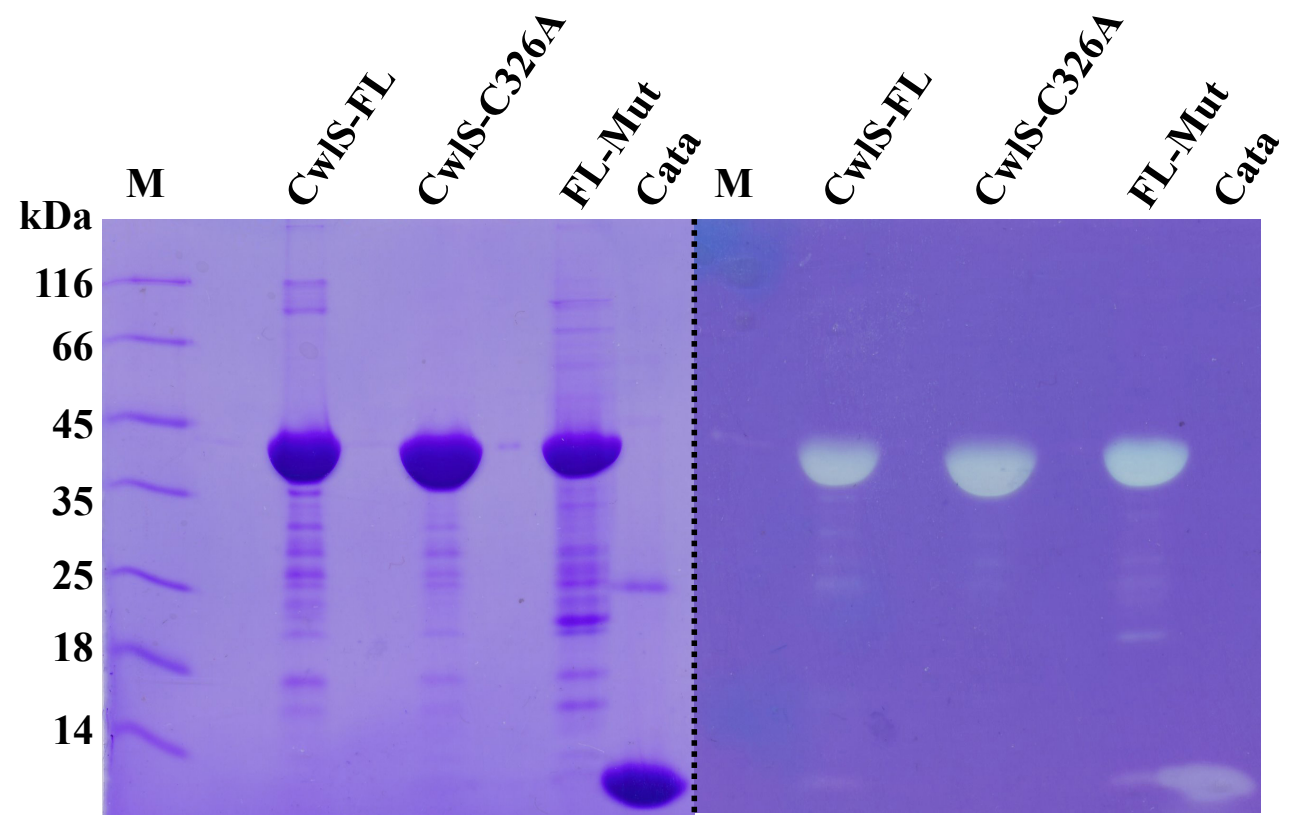

Coomassie stained 